\title{
Labyrinthe
}

17 | 2004 (1)

Jacques Rancière, l'indiscipliné

\section{Roman artiste et philosophie}

\section{Yvan Pineault}

\section{OpenEdition}

Journals

Édition électronique

URL : http://journals.openedition.org/labyrinthe/193

DOI : $10.4000 /$ labyrinthe.193

ISSN : 1950-6031

Éditeur

Hermann

Édition imprimée

Date de publication : 15 avril 2004

Pagination : 131-133

Référence électronique

Yvan Pineault, «Roman artiste et philosophie », Labyrinthe [En ligne], 17 | 2004 (1), mis en ligne le 17 juin 2008, consulté le 22 septembre 2020. URL : http://journals.openedition.org/labyrinthe/193 ; DOI https://doi.org/10.4000/labyrinthe.193

Propriété intellectuelle 


\title{
Roman artiste et philosophie
}

\author{
Yvan Pineault \\ brutewillis@aol.com
}

\begin{abstract}
Établir des comparaisons éthiques et esthétiques entre le roman dit « artiste » - ou « européen », dans la perspective de Milan Kundera ${ }^{1}$ et la philosophie écrite: tel est l'objet du présent commentaire, que l'on justifiera par la nécessité de déplacer l'état des réflexions portant sur les rapports entretenus par la littérature et la philosophie. L'idée d'une parenté fondamentale entre ces deux champs n'est, en effet, plus à démontrer; mais la recherche a, jusqu'à présent, concentré ses principaux efforts sur la sphère de la poésie, laissant hors du domaine les représentations narratives. Texte d'élargissement de la problématique donc, épousant l'éventualité d'une perspective descendante de la philosophie par rapport au roman « artiste » en matière d'esthétique et de questionnements éthiques.
\end{abstract}

\section{Esthétique du texte philosophique, du roman « artiste »}

C'est Kant qui, dans Critique du jugement, libère l'esthétique des référentiels et des modèles absolus : le jugement de goût devient subjectif, sans concept. Mise à plat de la tutelle exercée depuis Platon par la philosophie ? Non : l'esthétique demeure dans la perspective kantienne - et plus largement dans celle de tous les philosophes abordant la question de l'esthétique à partir du langage constitué - une discipline proprement philosophique: systématisée par la philosophie, elle est également du domaine du texte philosophique, édifié en une source de plaisir esthétique. Prolégomènes intéressés de la philosophie,

1 « Pour délimiter avec exactitude l'art dont je parle, je l'appelle roman européen. Je ne veux pas dire par là : romans créés en Europe par des Européens, mais : romans faisant partie d'une histoire qui a commencé à l'aube des Temps modernes en Europe » (Milan Kundera, Les Testaments trahis, Paris, Gallimard, 1993, p. 40); des territoires de sagesse, cherchant à élucider l'homme et sa place dans l'univers; des lieux où le jugement moral est donc suspendu, où toute connaissance peut être embrassée, mais aussi « dédivinisée » - ainsi, par exemple, dans Joseph et ses frères, la tétralogie de Thomas Mann, l'ordre de la Création renversé: à la divinité soufflant sur de la glaise, succède l'homme créateur, Abraham, qui choisit son Dieu parmi d'autres divinités: « Le Chaaldéen était l'homme qui était l'homme qui avait découvert Dieu. Dieu, dans sa joie, s'était baisé la pointe des doigts en s'écriant: 'Jusqu'à ce jour, nul humain ne m'avait appelé Seigneur et Tout-Puissant; c'est ainsi qu'on me nommera désormais' » (Thomas Mann, Le Jeune Joseph, Paris, Gallimard, coll. «L'imaginaire », 1980, p. 37). Le roman européen, artiste dans notre perspective, est un prolégomène du renouvellement de l'art et de la pensée ainsi que de leur déplacement; une profanation de ce qui est hiératique, absolu, statique. 
tentant de ramener l'art et le langage conceptuel à la même activité. Or, en ordonnant les principes de l'activité scripturale autour de la mise en place du discours conceptuel, c'est l'esthétisation même du texte philosophique qui s'en trouve ruinée. Métamorphosée en méthode philosophique, celui-ci n'exprime plus que les besoins d'un raisonnement métaphysique, et sa finalité. Limite esthétique fixée par la philosophie per se; limite esthétique en dehors de laquelle la philosophie deviendrait impraticable, son essence étant constituée de substrats conceptuels, exigeant des systèmes de contraintes et d'oppositions pour s'actualiser en tant que principes universels. Assujettie aux systèmes et aux concepts médités, toute esthétique est ainsi minée: l'écriture, l'ordre de la réflexion, les métaphores sont surdéterminés $a b$ ovo par des concepts concourants, motifs d'une modélisation du canal en fonction de leur mise en lumière. Démarche coercitive du philosophe à l'égard de lui-même, tentant de s'attribuer ce qu'il désigne, mais se restreignant dans la prolifération de sa pensée, donnant tout ce qu'il en reste à la création d'une intersection, d'un point de convergence où tout finit par avoir un sens, cela va de soi.

Par opposition, le roman « artiste » relève principalement d'un au-delà (ou d'un en deçà) des concepts: influence du romantisme, dont le concours ne se limite pas à une légitimation de l'expérience du moi. Le romantisme retranche en effet du domaine de la philosophie la mission de penser et d'ordonner la littérature de manière exclusive. Résultat: de nouveaux principes littéraires, articulés par ceux-là mêmes qui écrivent la littérature, substituant la liberté dans l'art aux règles déterminées pas le classicisme et la philosophie. Le renversement est significatif, et le roman en est le véhicule par excellence: jusqu'alors oublié de toutes les poétiques, il n'a, à la différence des autres genres littéraires, à s'affranchir d'aucune législation; il est, d'entrée de jeu, l'espace de liberté éthique et esthétique convoité. Émancipé des contraintes du texte philosophique, le roman, plus spécifiquement le roman « artiste », tend dès lors à ramener le langage conceptuel à un choix artistique : il est d'abord question de créer, de déployer, en tant qu'art, de nouvelles voies. Glissant hors des espaces consacrés, il explore toutes les pistes, déplace, fissure, rapporte en lui-même des fragments; il repousse les conventions de forme, les clôtures qui balisent les genres littéraires, s'accaparant leurs structures, irréductible aux limites, y compris les siennes. Car il est aussi son propre contestant: ses innovations sont aussi auto-transgressions, rotations du genre en un non-genre, soustrait aux typologies univoques.

\section{Éthique du texte philosophique, du roman « artiste »}

Libéré des contraintes du texte philosophique, le roman « artiste » s'impose comme un point où cesse le pouvoir, un espace d'innovations esthétique et éthique. Cependant que la philosophie constitue une réflexion utilisant des 
catégories qu'elle ne crée pas systématiquement, mais qu'elle récupère dans sa propre histoire, le roman « artiste » dévoile incessamment de nouvelles possibilités: en mouvance parmi les ruines où tout a été centré, recroquevillé en épistémè, il se déplace d'une réflexion à l'autre, exerçant son privilège de poser des questions que la philosophie, cantonnée dans une finalité conceptuelle, évite. La philosophie, traversée par le désir de poser des questions qui s'adressent à tous, est en effet impuissante devant tout ce qui, dans le monde, relève d'une expérience impartageable; limite éthique posée par la philosophie per se, tentant de tout ramener à une réglementation univoque. Inversement, parce qu'il ne tient pas compte d'une vérité universalisée à tout et à tous, le roman « artiste » parvient, plus que la philosophie, à dupliquer le monde selon toutes ses possibilités. Conscient que le monde se modifie, il le modifie en son sein, transgressant la temporalité, les mœurs et, de manière générale, toutes les réalités. Dans le roman " artiste », ce ne sont pas les concepts qui portent en eux une réflexion sur la condition humaine et la réalité, mais les pestiférés (La Peste d'Albert Camus), les enfants assassins (Le Grand Cahier d'Agota Kristof) et déformés (Cent Ans de solitude de Gabriel García Márquez), les tueurs mélomanes (Orange mécanique d'Antony Burgess); des silhouettes sur le papier, modélisées sur des réalités enfouies, dissimulées, ramenées à la lumière par le roman « artiste »; de faux existants, dont les figures sèment un doute sur les apparences du réel, et en donnent de nouvelles versions, hors de la caverne.

S'il est entendu que la philosophie s'affaire à tracer le possible de la pensée, le roman «artiste » en trace donc, d'une certaine manière, l'impossible, poussant au-delà du monde des possibles, tirant du néant de nouvelles possibilités pour la pensée. Un désastre, au sens où l'entendait Maurice Blanchot, désorientant tout absolu, cherchant à valider un autre langage. Or, dans le cadre de nos préoccupations, quelle est la nature fondamentale de l'absolu? Dans le roman: la préséance de la fiction que l'on maintient sur les concepts. Dans le texte philosophique : le concept et son autorité, que l'on tente de préserver d'une contamination de la fiction. Et si, par conséquent, le texte philosophique perdait toute raison d'être? 